Research Article

\title{
Evaluation of Sperm Impairing Factor from Serratia marcescens as Male Contraceptive in Mouse Model
}

\author{
Aditi Chauhan and Vijay Prabha \\ Department of Microbiology, Panjab University, Chandigarh-160014, India \\ Correspondence should be addressed to Vijay Prabha; satishvijay11@gmail.com
}

Received 3 June 2019; Revised 3 September 2019; Accepted 6 September 2019; Published 30 October 2019

Academic Editor: Abdelwahab Omri

Copyright (c) 2019 Aditi Chauhan and Vijay Prabha. This is an open access article distributed under the Creative Commons Attribution License, which permits unrestricted use, distribution, and reproduction in any medium, provided the original work is properly cited.

\begin{abstract}
The present study was carried out to assess the contraceptive efficacy of sperm agglutinating factor (SAF) isolated from Serratia marcescens, in male Balb/c mice. Mice were administered via an intratesticular route with different concentrations of SAF, viz., 10, 50, 100, 200, or $400 \mu \mathrm{g}$, in the right testis only which served as a test while the left side served as control except otherwise stated. Mice were sacrificed on day 3, 7, 14, 21, 30, 45, 60, and 90 after administration, and results in terms of change in body weight, seminal parameters, tissue somatic indices (TSI), hematological parameters, serum level of testosterone, lipid peroxidation, and histology were studied. The body weight and TSI remained unaffected in all the experimental groups. In case of seminal parameters, the right testis treated with $10 \mu \mathrm{g}, 50 \mu \mathrm{g}, 100 \mu \mathrm{g}, 200 \mu \mathrm{g}$, or $400 \mu \mathrm{g}$ of SAF showed azoospermia up to day $7,14,21,45$, and 90, respectively. The hematological indices, aspartate aminotransferase (AST), and alanine aminotransferase (ALT) were found to be unaltered when the group receiving SAF (test) was compared with the groups receiving phosphate buffer saline (control) in the right testis; however, the treatment had a negative effect on the serum level of testosterone. It also affected the oxidative status of the right testis. Furthermore, histological studies revealed hypospermatogenesis and alterations in the seminiferous tubules which included intraepithelial vacuolation and exfoliation in the right side as compared to the left side. Thus, the results suggest that SAF ( $400 \mu \mathrm{g}$ ) causes suppression of spermatogenesis, without causing apparent toxic effects.
\end{abstract}

\section{Introduction}

The world's population is now odds-on to grow ever-higher for the rest of the century, presenting grave difficulties for food supplies, healthcare, and social cohesion. Contraception is considered to be the key answer to this. Although commendable progress has been made in developing highly efficient, user-friendly, safe, and reversible contraceptive methods for females, progress in case of males is creeping at a slow pace. Surprisingly, even today, the contraceptive choices available for men do not differ much from the ones available in the first century. Until today, condom and vasectomy have been the major methods employed for contraception in males. While investigating other promising methods for male contraception, several methods have been identified using hormonal approaches. With various lacunae in these approaches, the challenge remains to come out with the compounds that are broadly acceptable, safe, cost-effective, and reversible. In this regard, some plants, as well as chemical compounds, have been recognized for their value in the field of contraception. These compounds led to hypospermatogenesis via several effects, viz., decrease in the testosterone level, disruption of the seminiferous tubules, and an increase in the oxidative stress. Though the intake of antioxidants may swamp the effects generated by these compounds [1-5], all other mechanisms contiguously affect the reproductive potential. The global scenario is now directing towards the use of alternative approaches which offer reversibility, efficacy, and safety with minimal side effects. So, the time has come to exploit the rich diversity of microorganisms since very little work has been done on their plausible application in 
this area. In the light of this initiative, previously in our laboratory, Serratia marcescens was found to produce a factor with sperm agglutinating property in vitro and showed admirable contraceptive efficacy in female mice [6]. These encouraging findings paved the way to extrapolate the same in male mice as a method of contraception. Also, due to the personal, productivity, and societal toll taken by surgery, a dire need continues to exist for nonsurgical methods of fertility control that are consistent, affordable, and satisfactory. Henceforward, the present study was designed for evaluating the impact of the sperm agglutinating factor (SAF) on the reproductive potential of male mice via an intratesticular route of administration.

\section{Materials and Methods}

2.1. Experimental Animals. Sexually mature male Balb/c mice (5-6 weeks old, $25 \pm 2 \mathrm{~g}$ ) were used in the present study, and they were housed in polypropylene cages. The animals were fed with standard pellet food and water ad libitum, and standard laboratory conditions (12:12, dark: light cycle) were retained. All the experimental work has been executed in accord with the procedures affirmed by Institutional Animal Ethics Committee, Panjab University vide letter no. PU/IAEC/S/16/140. All the experiments were completed in concurrence with the guidelines of the Committee for the Purpose of Control and Supervision of Experiments on Animals (CPCSEA).

2.2. Microorganism. A standard strain of Serratia marcescens (S. marcescens) (MTCC-7641), causing sperm agglutination in vitro, was used in the present study [6].

2.3. Isolation and Purification of the Sperm Agglutinating Factor (SAF) from $S$. marcescens. The sperm agglutinating factor was isolated and purified from a $72 \mathrm{~h}$ old cell culture of $S$. marcescens by the method earlier standardized in the laboratory [6].

2.4. Matrix-Assisted Laser Desorption/Ionization Time of Flight (MALDI-TOF) of SAF. Processing of protein band, tryptic digestion, and peptide extraction were performed as described by Gupta and Prabha [7]. Sample for MALDITOF analysis was prepared using the dried droplet method. $1 \mu \mathrm{L}$ peptide solution (peptide extracts after tryptic digestion) and $1 \mu \mathrm{L}$ of a suitable matrix, for example, alphacyano hydroxycinnamic acid (HCCA) in $1: 2 \mathrm{v} / \mathrm{v}$ of acetonitrile $(\mathrm{ACN}): 0.1 \%$ TFA, were mixed nicely. $1 \mu \mathrm{L}$ of this mixture was spotted on a MALDI target plate and allowed to air dry at room temperature. Peptide calibration standard (BRUKER) was also prepared in the same way. The MALDI target plate was loaded into Ultraflex MALDI-TOF for subsequent peptide spectra acquisition and analysis. Laser power of $337 \mathrm{~nm}$ wavelength was used for ionization of the samples spotted on the target plate. Peptide peaks were calibrated with peaks obtained from the peptide calibration standard. After peptide spectra were obtained, MS analysis was carried out using Flex analysis software (v 2.2, BRUKER). Subsequent MS data analysis was carried out using Biotools software ( $\mathrm{v}$ 2.2, BRUKER) and MASCOT search engine (Matrix Science) against the NCBI database.

2.5. Intratesticular Inoculation Procedure. Under surgical conditions, male Balb/c mice were inoculated with different concentrations of SAF, viz., 10, 50, 100, 200, or $400 \mu \mathrm{g}$, in the right testis under anesthesia of ketamine and xylazine. The scrotal skin was gently washed with saline solution and disinfected with isopropyl alcohol. The inoculum $(20 \mu \mathrm{l})$ was instilled into the right testis serving as test while the left testis served as control in all the experimental groups except in case of hematological parameters and serum level of testosterone, where mice administered with PBS in the right testis served as control in comparison to mice receiving SAF in the right testis which served as test. To check the effect of SAF, the animals were euthanized on day $3,7,14,21,30,45$, 60 , and 90 . The parameters evaluated include body weight profile, TSI (\%), seminal parameters, hematological parameters, serum level of testosterone, lipid peroxidation, and histological changes.

2.6. Weight Profile and Tissue Somatic Indices (TSI \%). The body weight of mice from each group on the $1^{\text {st }}$ day of the experiment was considered as initial body weight, and the body weight of the mice on the last day of the experiment was considered as final body weight. Mice were euthanized by cervical dislocation on the respective day of sacrifice. Mice were necropsied and various reproductive and nonreproductive organs were removed and weighed aseptically. The TSI (\%) (organ weight/body weight $\times 100$ ) of reproductive (vas deferens, testes, and cauda) and nonreproductive (kidneys, liver, spleen, and bladder) organs were estimated.

\subsection{Evaluation of Seminal Parameters}

2.7.1. Total Sperm Count. Three mice from each group were sacrificed on the respective day of sacrifice. Immediately after necropsy, each vas deferens from both sides (i.e., right (inoculated) side and left (uninoculated) side) was pulled out, placed in two glass plates containing freshly prepared, prewarmed $200 \mu \mathrm{l}$ of PBS ( $50 \mathrm{mM}, \mathrm{pH} 7.2$ ), and spermatozoa were enabled to swim out into the PBS by mild teasing. Furthermore, $10 \mu \mathrm{l}$ of the sample was placed on a clean glass slide and analyzed under a light microscope at 400x magnification. Around eight to ten fields were examined, and the mean number of spermatozoa in each field was multiplied by $10^{6}$.

2.7.2. Sperm Motility. $10 \mu \mathrm{l}$ of the semen sample was placed on a warm slide and analyzed (400x) under a light microscope. The motile and nonmotile sperms were counted in eight to ten fields, and percentage of motile sperms was determined. 
2.8. Hematological Parameters. Mice were divided into two groups, viz., test group (receiving $400 \mu \mathrm{g} \mathrm{SAF}$ ) and control group (receiving PBS), with 3 mice in each group. Mice from each group were sacrificed on day 90, and blood samples from mice in both the groups were analyzed for red blood cells (RBCs) and white blood cells (WBCs) counts, hemoglobin (hb) concentration, and hematocrit. Serum was analyzed to measure the activities of alanine aminotransferase (ALT) and aspartate aminotransferase (AST) according to the method of Reitman and Frankel [8].

2.9. Testosterone Assay. Mice from both the control group (receiving PBS) and the test group (receiving $400 \mu \mathrm{g}$ SAF) were sacrificed on day 90 , and serum level of testosterone was measured by ELISA using a commercial kit according to the manufacturer's directions. The sensitivity of the assay was $5 \mathrm{pg} / \mathrm{ml}$ with intra- and interassay coefficient of variations being $5.1 \%$ and $7.5 \%$, respectively.

2.10. Lipid Peroxidation. Mice receiving $400 \mu \mathrm{g}$ SAF in the right testis were sacrificed on day 3 and 7 , and the level of lipid peroxidation was determined by the method of Ohkawa et al. [9]. Briefly, $3.3 \mathrm{ml}$ TBA reagent $(0.2 \mathrm{ml}$ of $8.0 \%$ SDS, $1.5 \mathrm{ml}$ of $20 \%$ acetic acid, and $1.5 \mathrm{ml}$ of $0.8 \%$ aqueous thiobarbituric acid and $0.1 \%$ of butylated hydroxyltoluene) was mixed with $0.2 \mathrm{ml}$ tissue supernatant obtained after the homogenization of testes, and the mixture was boiled at $95^{\circ} \mathrm{C}$ in a water bath for $60 \mathrm{~min}$. The solution was cooled and centrifuged at $2000 \mathrm{rpm}$ for $10 \mathrm{~min}$. The supernatant was then used for recording absorbance against blank (distilled water) at $532 \mathrm{~nm}$.

2.11. Histological Analyses. Mice from each group receiving a different concentration of SAF $(10,50,100,200$, or $400 \mu \mathrm{g})$ were sacrificed on day $3,7,14,21,30,45,60$, and 90 . The reproductive and nonreproductive organs after extraction were evacuated and fixed in $10 \%$ formaldehyde for $24 \mathrm{~h}$. After $24 \mathrm{~h}$, the tissues were inserted in paraffin. The paraffin tissue segments of $4 \mathrm{~mm}$ were stained with hematoxylineosin. Furthermore, slides were seen at 400x magnification for any noteworthy changes.

\section{Results}

3.1. Isolation and Purification of SAF from S. marcescens. Isolation and purification of SAF were performed by the method already standardized in our laboratory. Briefly, SAF was precipitated with $60-80 \%$ saturation of ammonium sulphate. After dialysis, it was subjected to Sephadex G-200 column, and from column chromatographic pattern, it was observed that the agglutinating activity was present in the fractions 4-8 with a peak value in fraction 5. Furthermore, the bioactive fractions were pooled, concentrated, and applied to a DEAE cellulose column. The column was run using a step gradient of $\mathrm{NaCl}$, and SAF could be eluted with PBS containing $0.6 \mathrm{M} \mathrm{NaCl}$. The molecular weight of purified
SAF obtained after ion-exchange chromatography was estimated to be $\sim 54 \mathrm{kDa}$ by SDS-PAGE.

3.2. MALDI-TOF of Purified SAF. According to MALDITOF analysis of the purified $54 \mathrm{kDa}$ band, the protein showed the peptide sequence similarity with adenylate kinase when the resulting spectrum was used to search for matching proteins in the NCBInr database, using the Mascot search program. The search yielded a top score of 56 for adenylate kinase (Figure 1).

3.3. Weight Profile. Single unilateral administration of different concentrations of SAF revealed no statistically significant changes in the body weight profile of male mice in all the groups (Supplementary Figure 1).

3.4. Tissue Somatic Indices (TSI \%). The TSI of reproductive and nonreproductive organs excised from all the mice receiving different concentrations of SAF were determined on all the days of sacrifice and the results so obtained indicated no significant changes in the TSI of all the organs (Supplementary Figures 2 and 3).

3.5. Evaluation of Seminal Parameters. In order to assess treatment-related changes in seminal parameters, mice $(n=3)$ were euthanized on the respective day of sacrifice and sperm count and motility were assessed, and it was found that unilateral administration of SAF had sperm impairing effects on the right side of treatment only, whereas the left side showed no changes in seminal parameters. In the case of mice treated with $10 \mu \mathrm{g}$ of SAF, it was observed that the right side displayed azoospermia which persisted till day 7 only, whereas the left side displayed normal sperm count. Day 7 onwards the sperm count started to restore and on day 14 there was only a $20 \%$ decrease in the sperm count in the right side in comparison to the left side. Furthermore, on day 21 the sperm count of both the sides was comparable. Since complete inhibition of spermatogenesis on day 3 and 7 was observed, motility could not be assessed for the same, while on day 14 and 21 the motility in the right side was comparable to the left side (Table 1). In case of mice treated with a higher concentration of SAF, i.e., $50 \mu \mathrm{g}$, azoospermia persisted in the right side up to day 14, whereas the left side showed normal sperm count. Thereafter, the sperm count started to restore, and on day 21 in the right side, there was only an $18 \%$ decrease in the sperm count in comparison to the left side. Furthermore, on day 30 , the sperm count in the right side was comparable to the left side. In the case of motility, until day 14, motility could not be assessed due to complete inhibition of the spermatogenesis in the right side, while, on day 21 , on the right side a $7 \%$ decrease was observed with respect to the left side, and it was comparable on day 30 (Table 1).

Mice treated with $100 \mu \mathrm{g}$ of SAF showed azoospermia in the right side till day 21 with respect to the left side which 


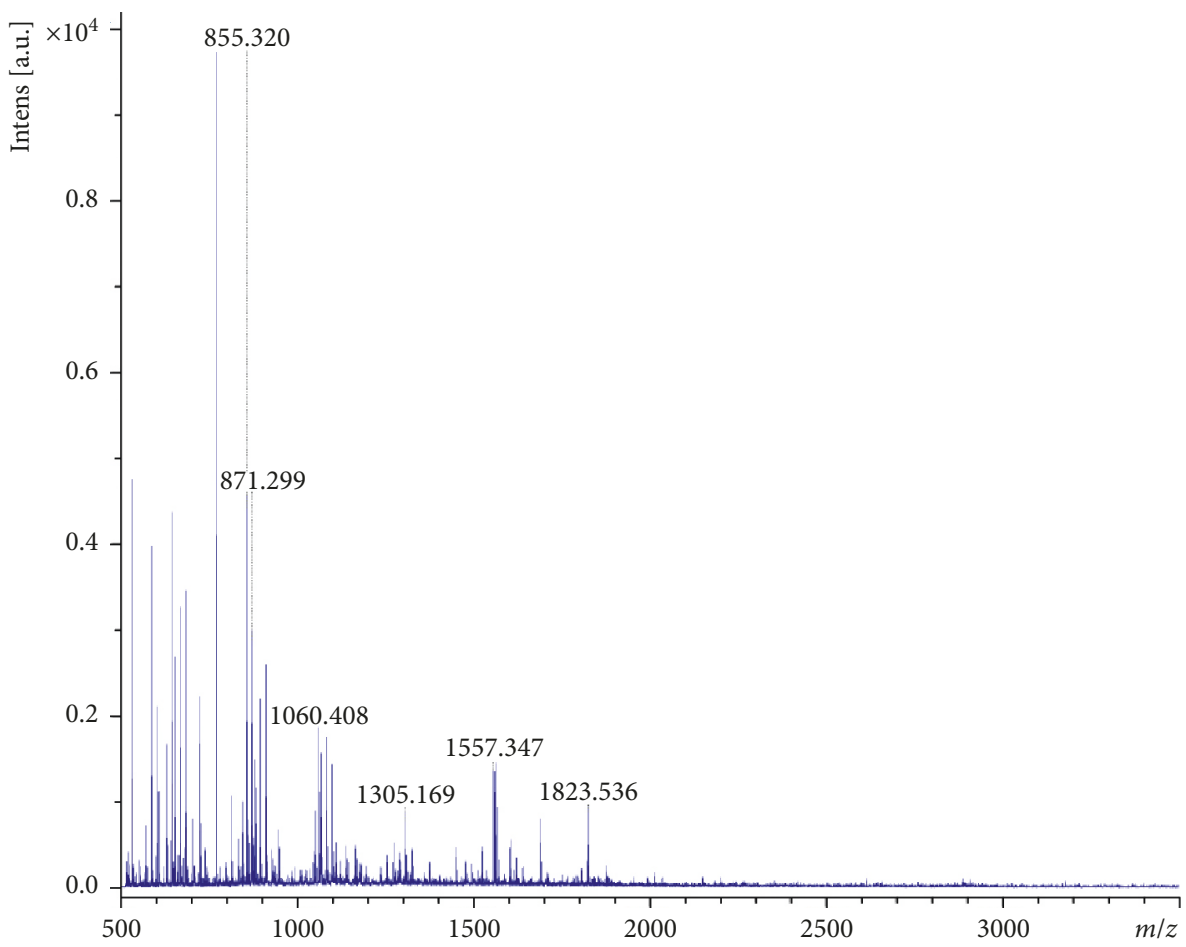

Figure 1: Mass spectrometric analysis of SAF after in-gel digestion with trypsin.

showed normal sperm count. On day 30, a $45 \%$ decrease in the sperm count was observed compared to the left side and sperm count started to restore thereafter, and on day 45 sperm count of the right side was comparable to the left side. Motility could not be assessed till day 21 due to complete inhibition of spermatogenesis in the right side. On day 30 , a $30 \%$ decrease in the motility was observed on the right side in comparison to the left side (Table 1), and on day 45 motility of both sides was comparable. In case of mice treated with $200 \mu \mathrm{g}$, azoospermia persisted in the right side up to day 45 , whereas the left side showed normal sperm count. Thereafter, the sperm count started to restore, and on day 60 the sperm count on the right side was comparable to the left side. In the case of motility, until day 45 , motility could not be assessed due to complete inhibition of the spermatogenesis in the right side, while, on day 60 , motility was comparable on both sides (Table 1). With the concentration $400 \mu \mathrm{g}$ SAF, azoospermia could be achieved till day 90, whereas the left side displayed normal sperm count. Furthermore, on all the days, the motility could not be assessed due to complete inhibition of spermatogenesis in the right side (Table 1).

3.6. Hematological Parameters. No significant changes were found in the levels of AST $(32.19 \pm 3.12)$ and ALT $(24.22 \pm 0.45)$ in the treated mice compared to control mice AST $(29.01 \pm 3.01)$ and ALT $(26.34 \pm 0.19)$ in serum. Similarly, hematological parameters (haemoglobin, RBC, WBC, and hematocrit) in test (SAF $400 \mu \mathrm{g}$ ) were comparable to the control (PBS) throughout the course of investigation (Table 2).
3.7. Testosterone Assay. A marked decrease was noted in the serum testosterone level after intratesticular administration of SAF $(400 \mu \mathrm{g})$ in the test $(0.43 \pm 0.02)$ as compared to the control $(1.86 \pm 0.03)$ receiving PBS (Table 2$)$.

3.8. Lipid Peroxidation. Following the single unilateral administration of $400 \mu \mathrm{g} \mathrm{SAF}$, a significant increase in endogenous thiobarbituric acid reactive substance (TBARS) levels was observed on day 3 and 7 in the right side (562.94 $\mu$ moles/g of tissue and $511.97 \mu$ moles $/ g$ of tissue) as compared to the left side $(65.38 \mu$ moles/g of tissue and 74.65 $\mu$ moles/g of tissue) (Figure 2).

3.9. Histological Examination. To check any adverse impact of SAF on tissue morphology, histological analysis of different reproductive (testis, cauda epididymis, and vas deferens) and nonreproductive organs (spleen, kidney, bladder, and liver) was carried out. The right set of organs of mice treated with SAF $(400 \mu \mathrm{g})$ showed azoospermia with marked alterations in the histoarchitecture, viz., loosening and sloughing of germinal epithelial in testes (Figure 3(d)), caudal epididymis showed empty tubules (Figure 3(e)), while vas deferens showed normal tissue histology (Figure 3(f)). However, the left set of reproductive organs divulged normal tissue histology, i.e., testis showed regular germinal epithelium and seminiferous tubules with progressive phases of change of spermatogonia into spermatozoa (Figure 3(a)), epididymis displayed filled tubules (Figure 3(b)), and vas deferens showed normal columnar epithelium (Figure 3(c)), while all the nonreproductive 


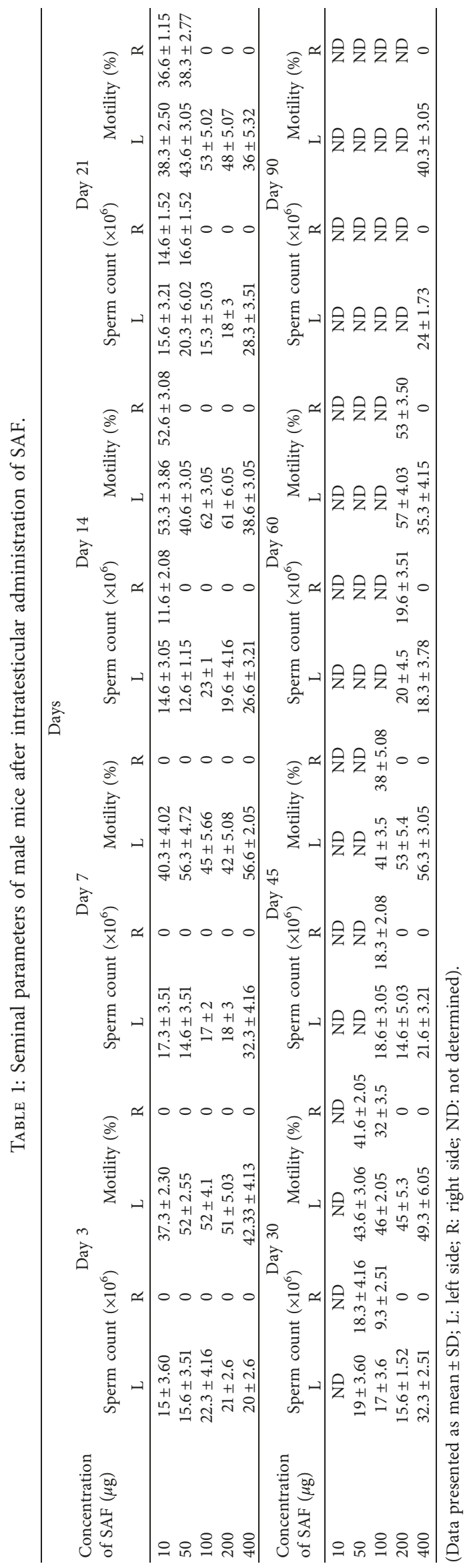


TABLE 2: Effect of intratesticular administration of SAF $(400 \mu \mathrm{g})$ on hematological parameters and hormonal levels of male mice.

\begin{tabular}{lcc}
\hline & Hematological parameters & \\
Parameters & Control (PBS) & SAF $(400 \mu \mathrm{g})$ \\
\hline $\mathrm{RBC}\left(10^{6} / \mathrm{mm}^{3}\right)$ & $4.54 \pm 0.21$ & $4.72 \pm 0.07$ \\
$\mathrm{WBC}\left(10^{3} / \mathrm{mm}^{3}\right)$ & $6.51 \pm 0.31$ & $6.02 \pm 0.15$ \\
$\mathrm{Hb}(\mathrm{g} / \mathrm{dl})$ & $13.4 \pm 0.02$ & $13.9 \pm 0.05$ \\
Lymphocytes (\%) & $78.16 \pm 0.03$ & $81.01 \pm 0.01$ \\
Monocytes $\%)$ & $7 \pm 0.18$ & $5 \pm 0.27$ \\
Neutrophils (\%) & $15 \pm 0.09$ & $13 \pm 0.19$ \\
Eosinophils (\%) & $0.08 \pm 0.04$ & $0.03 \pm 0.16$ \\
Basophils $(\%)$ & $0.04 \pm 0.21$ & $0.07 \pm 0.31$ \\
AST $(\mu / \mathrm{ml})$ & $29.01 \pm 3.01$ & $32.19 \pm 3.12$ \\
ALT $(\mu / \mathrm{ml})$ & $26.34 \pm 0.19$ & $24.22 \pm 0.45$ \\
\hline & Hormonal level & \\
Testosterone $(\mathrm{ng} / \mathrm{ml})$ & $1.86 \pm 0.03$ & $0.43 \pm 0.02$ \\
\hline
\end{tabular}

(Data presented as mean $\pm \mathrm{SD})$.

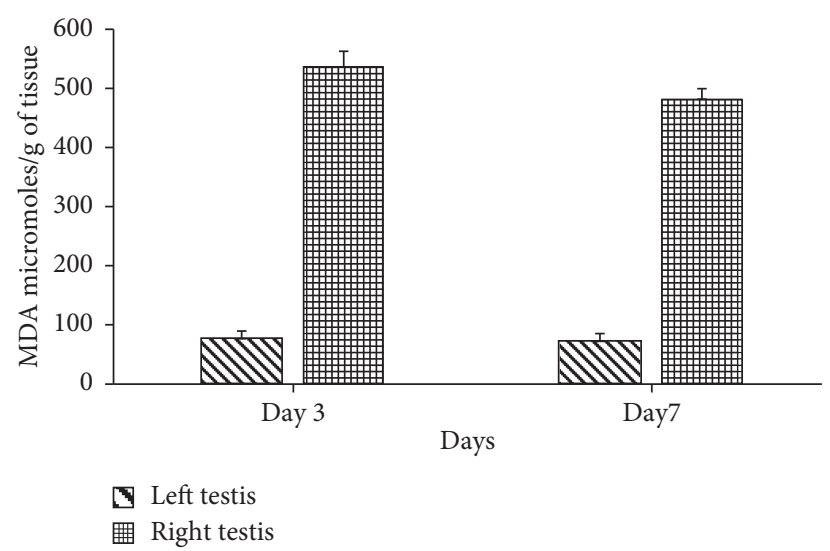

FIGURE 2: Lipid peroxidation in terms of MDA ( $\mu$ moles/g of tissue) of testis in the right side in comparison to the left side on day 3 and 7. Data represent mean values $\pm \mathrm{SD} .\left({ }^{*}\right),\left({ }^{* *}\right)$, and $\left({ }^{* * *}\right)$ represent $p<0.05, p<0.01$, and $p<0.001$, respectively.

organs were observed to be histologically normal in all the cases (Figure 4).

\section{Discussion}

In the present study, we have made an attempt to demonstrate the effect of SAF on the reproductive potential of male mice by monitoring its effect on changes in body weight, TSI, hematological parameters, seminal parameters, testosterone level, lipid peroxidation, and tissue histology. The body weight profile of SAF-treated mice showed no significant changes and these findings are in line with the study by Reddy et al. [10] and Chauhan and Agarwal [11]; wherein, no change was reported in the body weight of the male rats treated with a microbial peptide nisin and extract of Cassia fistula. Furthermore, evaluation of TSI (\%) of reproductive organs and nonreproductive organs was carried out to investigate their functional status ensuing various experimental conditions, and results of unilateral administration of SAF in right testis revealed no significant changes in \%TSI. Furthermore, there was no significant difference in the hematological parameters, ALT, and AST in the treated group in comparison to the control. These results are in concordance with the studies where the antifertility effect of the extracts of Coccinia indica, Mentha arvensis, and a protein isolated from the root of Achyranthes aspera were evaluated. Thus, the absence of any changes in body weight, \%TSI, and hematological parameters suggests the nontoxic nature of SAF and also indicates that it does not have an inimical effect on the general body metabolism.

Although various approaches for quantification of sperm quality have been suggested, seminal parameters have been most commonly used since high sperm count and motility are prerequisites for fertilisation and correlate strongly with fertilisation success. The results of the present study revealed complete loss of sperm count on the treated side after administration of $400 \mu \mathrm{g}$ of SAF. These results are in agreement with the studies carried out by Upadhyay et al. [12] who have shown that single unilateral administration of neem oil in male rats led to azoospermia in the side of treatment only.

There was a decrease in the testosterone level in the SAFtreated mice as compared to the control. It is well known that testosterone plays an important role in spermatogenesis and affects the epididymal milieu [13], so it must have affected the spermatogenesis. But, as hypospermatogenesis was seen only in the right side, there might be other factors playing an 

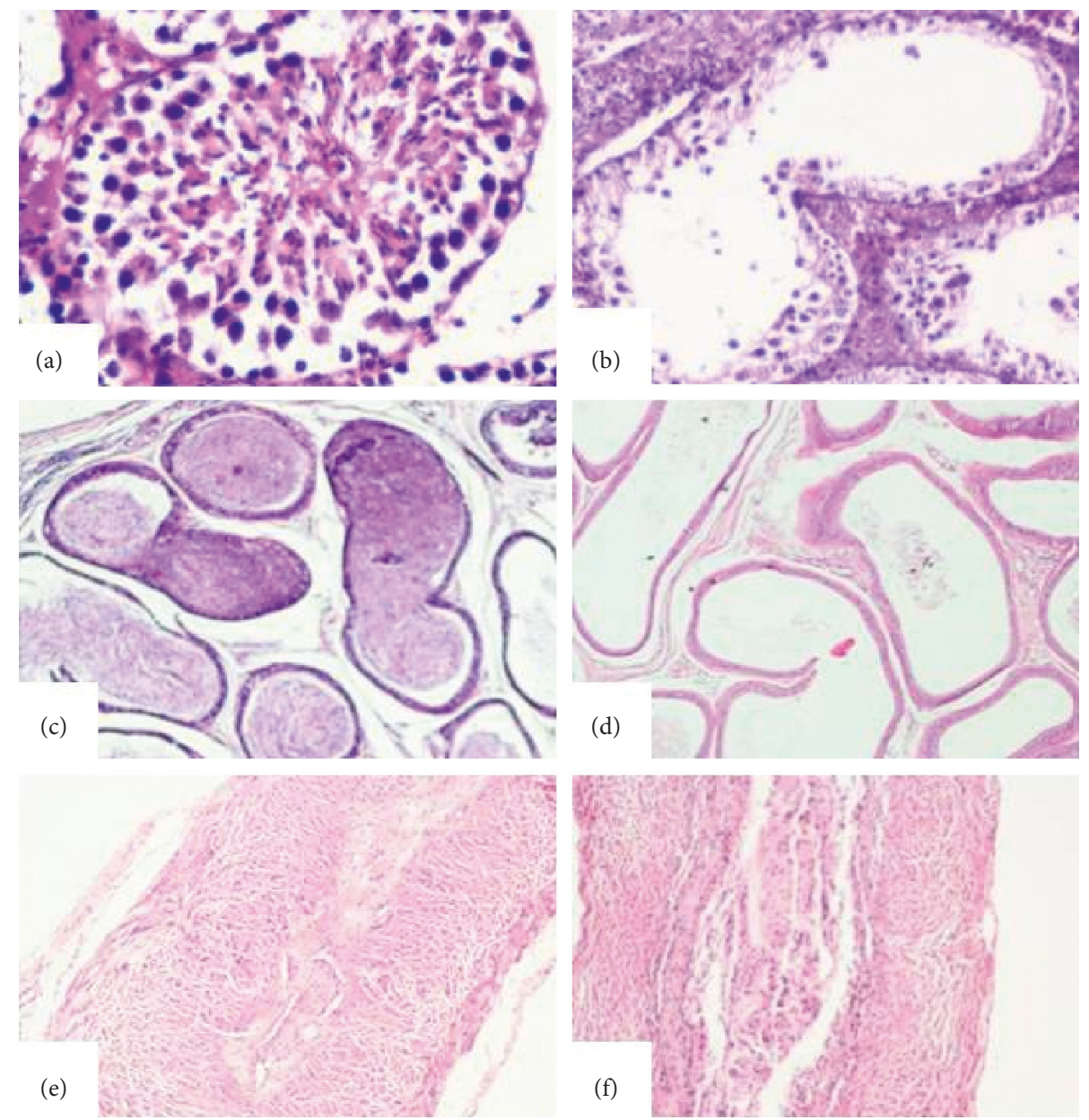

FIGURE 3: Representative photomicrographs of histological examination (day 90) of reproductive organs, viz., testis, cauda epididymis, and vas deferens of left side (a, b, and c, respectively) and right side (d, e, and f, respectively) receiving $400 \mu \mathrm{g}$ SAF. Left testis: (a) normal seminiferous tubules (indicated by arrow head); left cauda epididymis: (b) tubules filled with spermatozoa (indicated by arrow head); left vas deferens: (c) normal tissue histology (abbreviated as N). Right testis: (d) degenerative changes in the seminiferous tubules and empty testicular lumen (abbreviated as ETL); right cauda epididymis: (e) empty epididymal lumen (abbreviated as EEL); right vas deferens: (f) normal tissue histology (abbreviated as $\mathrm{N}$ ).

important role also. To understand the other possible mechanism by which SAF has affected the spermatogenesis, oxidative stress was checked as it is one of the most important factors affecting fertility by disturbing the spermatogenesis and steroidogenesis in testis. Due to the oxidative stress, free radicals are generated and these free radicals agitate the steroidogenic capacity of Leydig cells and differentiation of normal spermatozoa of germinal epithelium. Besides this, they also affect the cell membrane of spermatozoa because of the presence of a high amount of polysaturated fatty acids in their membrane, subsequently, prompting axonemal harm, decline in viability, and increase in morphological changes $[14,15]$. So, the rise in the level of lipid peroxidation in the treated testis as compared to the nontreated side in the present study implies that the oxidative stress conditions in the treated side might have also played a crucial role in inhibiting the spermatogenesis and steroidogenesis.

To verify the effects of SAF, tissue histology was undertaken, where the right testis and right epididymis showed inhibition of spermatogenesis and empty epididymal tubules as compared to the left side where normal tissue histology was observed. The right testis showed significant degenerative changes in the seminiferous tubules and the changes brought about by the SAF in seminiferous tubule were uniform. The changes observed in the seminiferous tubules were intraepithelial vacuolization, loosening of germinal epithelium, and exfoliation of the germ cells. These results are in accordance to earlier studies where similar results were obtained when male mice were treated with gossypol tetra acetic acid and the leaf extracts of Azadirachta indica, Allamanda cathartica, Curcuma longa, Dalbergia sissoo, and SC12937 [16-21]. These degenerative changes in the seminiferous tubules of the right testis can be correlated to the azoospermia observed on the right side only while investigating the seminal parameters. As the unilateral administration of SAF affected only the reproductive organs of right side, it can be assumed that the inhibition of spermatogenesis was not mediated by any systemic mechanism as it would have otherwise affected the organs of both sides. 

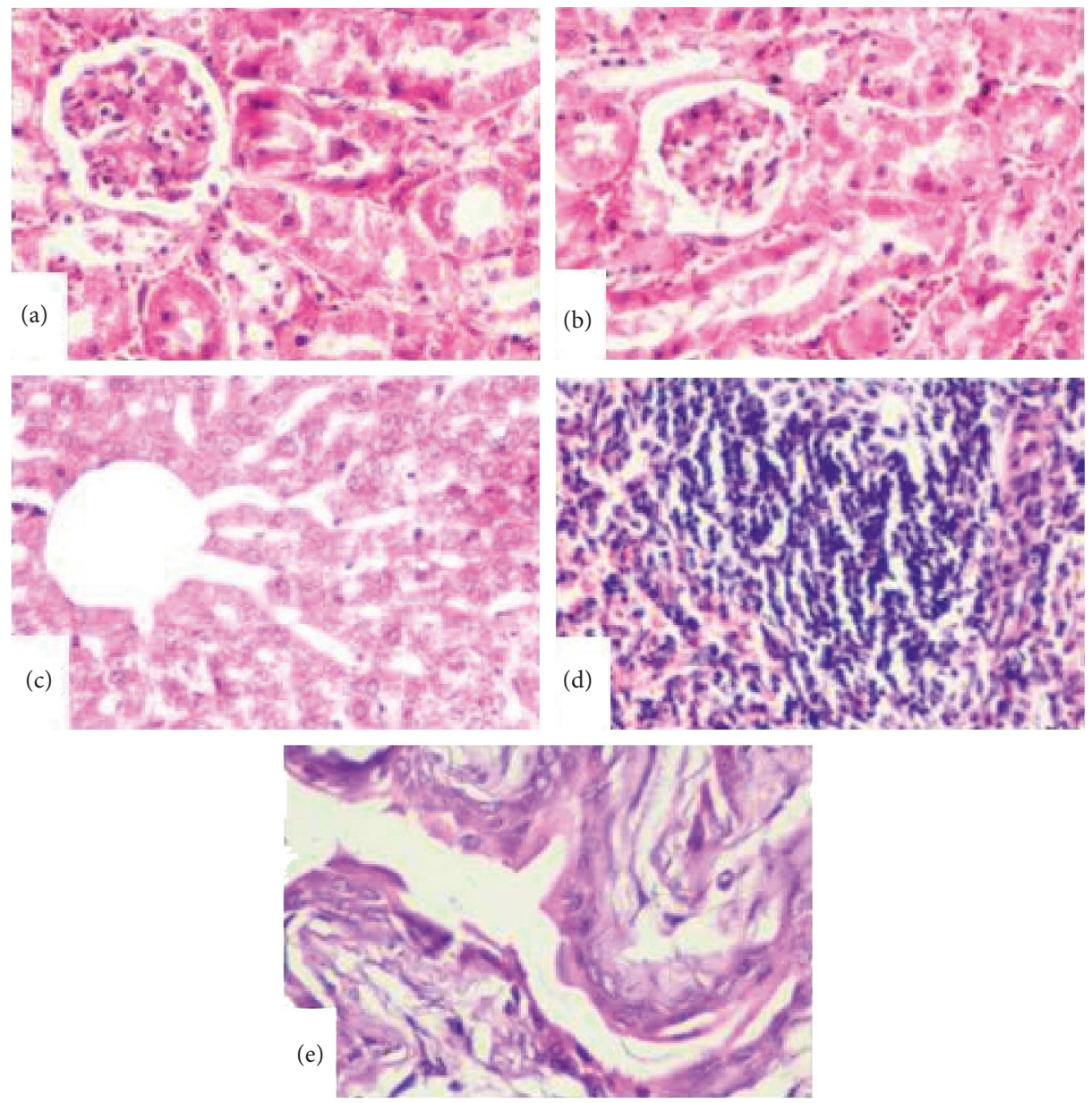

FIGURE 4: Representative histological photomicrographs (day 90) of various nonreproductive organs of male mice receiving $400 \mu \mathrm{g}$ SAF. (a) Left kidney. (b) Right kidney. (c) Liver (d) Spleen. (e) bladder.

In summary, intratesticular administration of SAF might be suppressing the spermatogenesis by affecting the steroidogenesis and by oxidative stress, which in turn leads to suppression of spermatogenesis, without eliciting any detectable toxic effects. Therefore, based on overall findings in the present study, it can be concluded that SAF holds the potential to be exploited as a male contraceptive in future.

\section{Data Availability}

The data used to support the findings of this study are included within the supplementary information files.

\section{Conflicts of Interest}

The authors declare that there are no conflicts of interest regarding the publication of this paper.

\section{Acknowledgments}

The authors thank the Department of Science and Technology, New Delhi, India (grant no. DST/Inspire Fellowship/2015/IF150184) for the funding and Dr. B. N. Dutta (Retd. Pathologist, PGIMER, Chandigarh, India) for the histological analysis.

\section{Supplementary Materials}

Supplementary Figure 1: body weight response of male Balb/ c mice inoculated intravasally with SAF: (a) $10 \mu \mathrm{g}$, (b) $50 \mu \mathrm{g}$, (c) $100 \mu \mathrm{g}$, (d) $200 \mu \mathrm{g}$, and (e) $400 \mu \mathrm{g}$. Supplementary Figure 2: tissue somatic indices (\%) of various reproductive organs of mice administered with SAF: (a) $10 \mu \mathrm{g}$, (b) $50 \mu \mathrm{g}$, (c) $100 \mu \mathrm{g}$, (d) $200 \mu \mathrm{g}$, and (e) $400 \mu \mathrm{g}$. Supplementary Figure 3: tissue somatic indices (\%) of various nonreproductive organs of mice administered with SAF: (a) $10 \mu \mathrm{g}$, (b) $50 \mu \mathrm{g}$, (c) $100 \mu \mathrm{g}$, (d) $200 \mu \mathrm{g}$, and (e) $400 \mu \mathrm{g}$. (Supplementary Materials)

\section{References}

[1] A. W. K. Yeung, N. T. Tzvetkov, O. S. El-Tawil, S. G. Bungau, M. M. Abdel-Daim, and A. G. Atanasov, "Antioxidants: scientific literature landscape analysis," Oxidative Medicine and Cellular Longevity, vol. 2019, Article ID 8278454, 11 pages, 2019.

[2] M. M. Abdel-Daim, K. Abo-EL-Sooud, L. Aleya, S. G. Bungau, A. Najda, and R. Saluja, "Alleviation of drugs and chemicals toxicity: biomedical value of antioxidants," Oxidative Medicine and Cellular Longevity, vol. 2018, Article ID 6276438, 2 pages, 2018. 
[3] M. M. Abdel-Daim, N. I. Zakhary, L. Aleya, S. G. Bungau, R. A. Bohara, and N. J. Siddiqi, "Aging, metabolic, and degenerative disorders: biomedical value of antioxidants," $O x$ idative Medicine and Cellular Longevity, vol. 2018, Article ID 2098123, 2 pages, 2018.

[4] M. M. Abdel-Daim, Y. M. Moustafa, M. Umezawa, K. V. Ramana, and E. Azzini, "Applications of antioxidants in ameliorating drugs and xenobiotics toxicity: mechanistic approach," Oxidative Medicine and Cellular Longevity, vol. 2017, Article ID 4565127, 2 pages, 2017.

[5] M. M. Abdel-Daim, S. M. Aly, K. Abo-el-Sooud, M. Giorgi, and S. Ursoniu, "Role of natural products in ameliorating drugs and chemicals toxicity," Evidence-Based Complementary and Alternative Medicine, vol. 2016, Article ID 7879406, 2 pages, 2016.

[6] V. Prabha, S. Negi, A. Chauhan, H. Vander, K. Rana, and D. Thaper, "Antifertility effect of sperm agglutinating factor isolated from Serratia marcescens: an in vivo study," Journal of Reproductive Endocrinology \& Infertility, vol. 3, p. 5, 2018.

[7] S. Gupta and V. Prabha, "Human sperm interaction with Staphylococcus aureus: a molecular approach," Journal of Pathogens, vol. 2012, Article ID 816536, 7 pages, 2012.

[8] S. Reitman and S. Frankel, "A colorimetric method for the determination of serum glutamic oxalacetic and glutamic pyruvic transaminases," American Journal of Clinical $\mathrm{Pa}$ thology, vol. 28, no. 1, pp. 56-63, 1957.

[9] H. Ohkawa, N. Ohishi, and K. Yagi, "Assay for lipid peroxides in animal tissues by thiobarbituric acid reaction," Analytical Biochemistry, vol. 95, no. 2, pp. 351-358, 1979.

[10] K. V. R. Reddy, S. M. Gupta, and C. C. Aranha, "Effect of antimicrobial peptide, nisin, on the reproductive functions of rats," ISRN Veterinary Science, vol. 2011, Article ID 828736, 8 pages, 2011 .

[11] A. Chauhan and M. Agarwal, "Evaluating the antifertility potential of an aqueous extract from Cassia fistula seeds in male rats," Fertility and Sterility, vol. 93, no. 5, pp. 1706-1710, 2010.

[12] S. N. Upadhyay, S. Dhawan, and G. P. Talwar, "Antifertility effects of neem (Azadirachta indica) oil in male rats by single intra-vas administration: an alternate approach to vasectomy," Journal of Andrology, vol. 14, no. 4, pp. 275-281, 1993.

[13] L. B. Smith and W. H. Walker, "The regulation of spermatogenesis by androgens," Seminars in Cell \& Developmental Biology, vol. 30, pp. 2-13, 2014.

[14] D. B. Hales, J. A. Allen, T. Shankara et al., "Mitochondrial function in Leydig cell steroidogenesis," Annals of New York Academy of Sciences, vol. 1061, no. 1, pp. 120-134, 2005.

[15] R. J. Aitken and S. D. Roman, "Antioxidant systems and oxidative stress in the testes," Oxidative Medicine and Cellular Longevity, vol. 1, no. 1, pp. 15-24, 2008.

[16] R. K. Mishra and S. K. Singh, "Effect of aqueous leaf extract of Azadirachta indica on the reproductive organs in male mice," Indian Journal of Experimental Biology, vol. 43, no. 11, pp. 1093-1103, 2005.

[17] S. Singh and S. Chakravarty, "Antispermatogenic and antifertility effects of 20,25-diazacholesterol dihydrochloride in mice," Reproductive Toxicology, vol. 17, no. 1, pp. 37-44, 2003.

[18] A. Singh and S. K. Singh, "Reversible antifertility effect of aqueous leaf extract of Allamanda cathartica L. in male laboratory mice," Andrologia, vol. 40, no. 6, pp. 337-345, 2008.

[19] S. K. Singh and S. K. Rath, "Effect of gossypol tetra acetic acid on the reproductive organs in male mice," in Recent Progress in Medicinal Plants, V. K. Singh and J. N. Govil, Eds., vol. 25, pp. 167-184, Studium Press, Houston, TX, USA, 2008.

[20] R. K. Mishra and S. K. Singh, "Reversible antifertility effect of aqueous rhizome extract of Curcuma longa L. in male laboratory mice," Contraception, vol. 79, no. 6, pp. 479-487, 2009.

[21] H. P. Verma and S. K. Singh, "Effect of aqueous leaf extract of Dalbergia sissoo Roxb. on spermatogenesis and fertility in male mice," The European Journal of Contraception \& Reproductive Health Care, vol. 19, no. 6, pp. 475-486, 2014. 


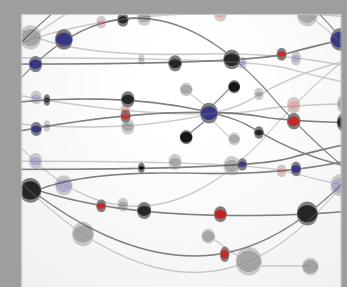

The Scientific World Journal
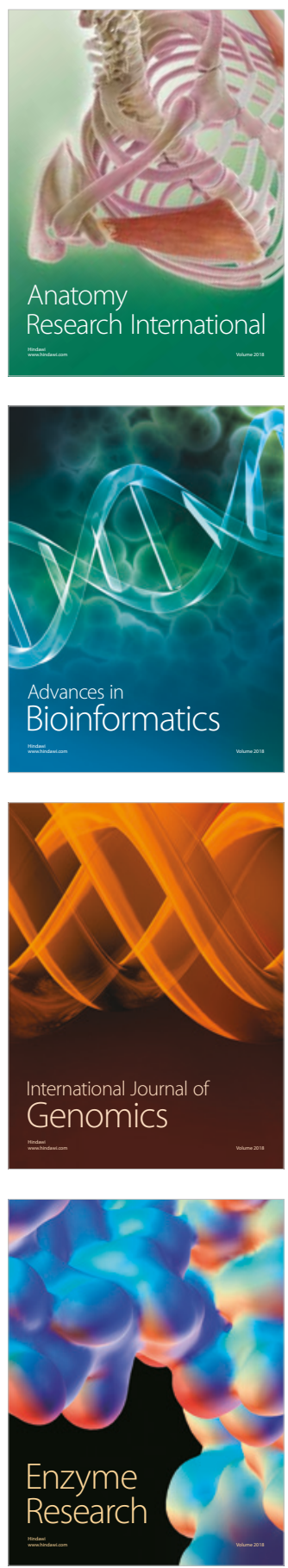
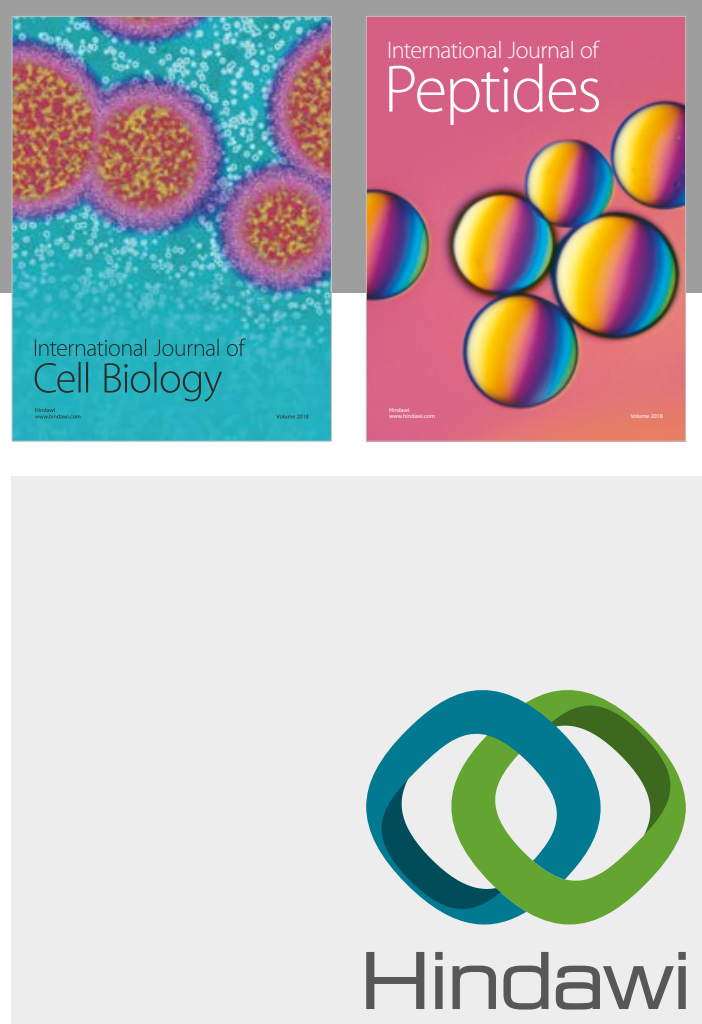

Submit your manuscripts at

www.hindawi.com
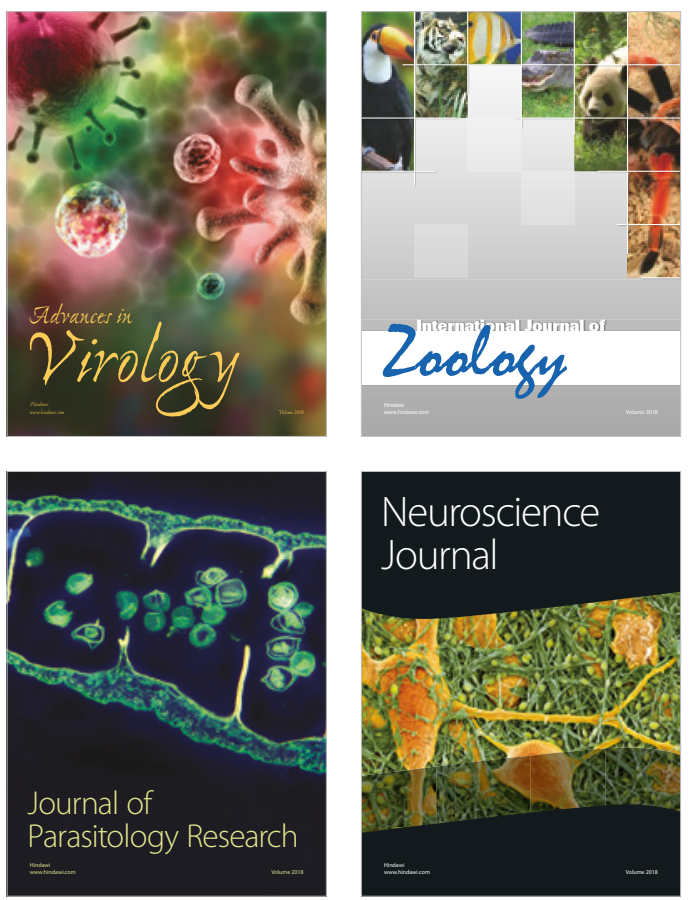
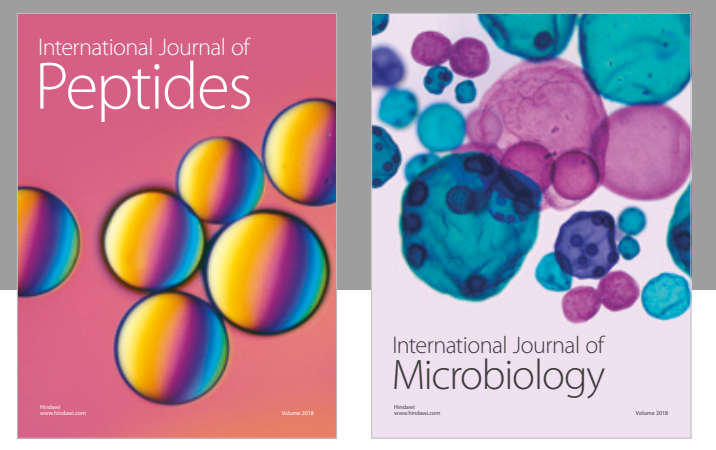

nternational Journal of Microbiology
Journal of
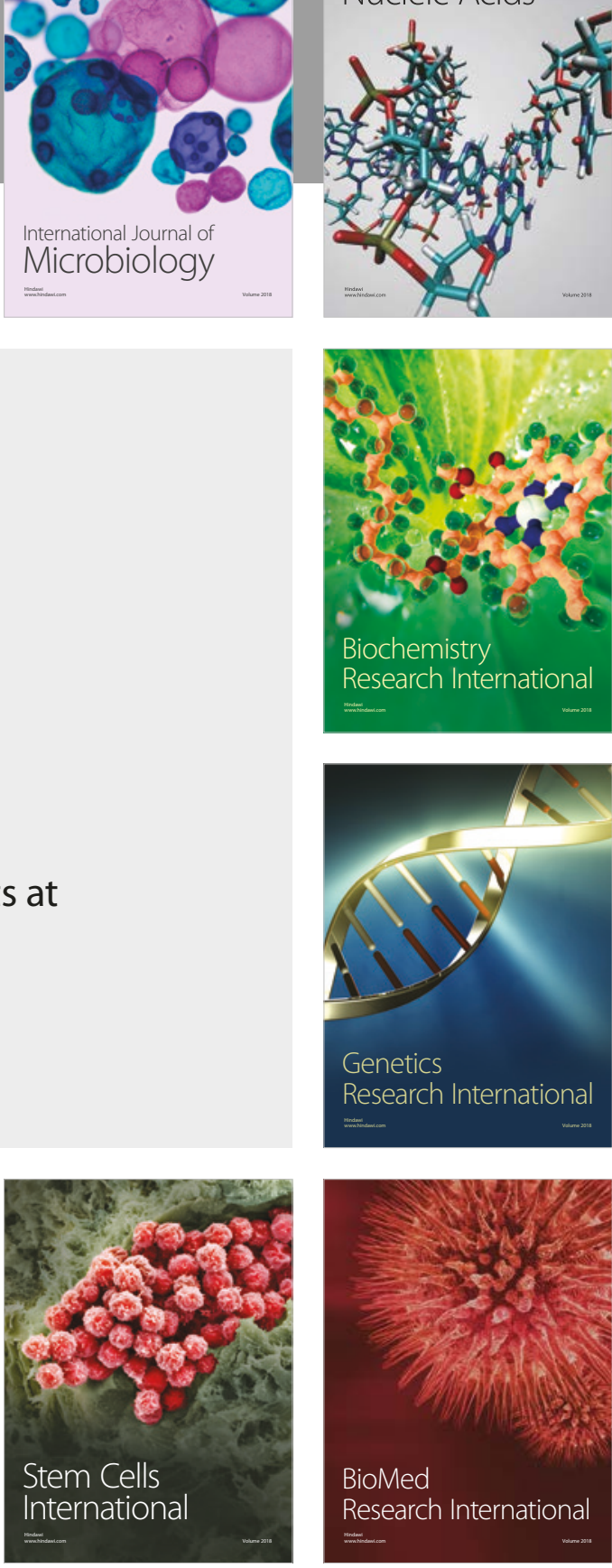
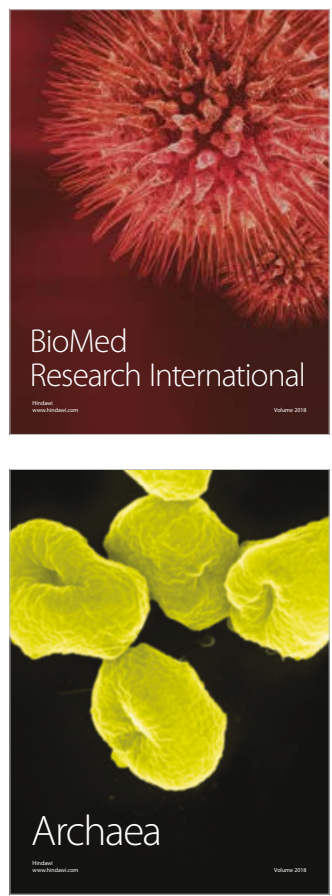